\title{
Special issue on advances in ambient intelligence and pervasive computing
}

\author{
Elhadi M. Shakshuki ${ }^{1} \cdot$ Ansar-UI-Haque Yasar ${ }^{2} \cdot$ Haroon Malik $^{3}$
}

Published online: 10 December 2019

○) Springer-Verlag GmbH Germany, part of Springer Nature 2019

This special issue is based on the best papers selected from the 10th International Conference on Ambient Systems, Networks and Technologies (ANT-2019), which was held on April 29-May 2, 2019, in Leuven, Belgium. The conference attracted a large number of scientific papers that contributed to the state-of-the-art in the ambient systems, networks and technologies. All the papers selected for this special issue have been extended significantly from their original versions and underwent two rounds of rigorous peer-review process. Based on the reviewers' feedback, as well as the evaluations of the Guest Editors, eight papers were selected for this special issue from 11 invited submissions. The accepted papers augment ambient systems by uncovering interesting methodologies related to bid data management, indoor localization, recommendation system with reinforcement learning, interference in wireless channels, and scheduling in cloud computing.

The first paper by Maguerra et al. is entitled "Towards a Reactive System for Managing Big Trajectory Data". The authors of this paper discussed the importance of extracting knowledge from heterogeneous, massive data generated from indoor and outdoor tracking devices. The main aim of this paper is to incorporate a fully-fledged, reactive system for big trajectory data management. The authors claim that this system is unique of its kind because it is actor-based

Elhadi M. Shakshuki

elhadi.shakshuki@acadiau.ca

Ansar-Ul-Haque Yasar

ansar.yasar@uhasselt.be

Haroon Malik

malikh@marshall.edu

1 Jodrey School of Computer Science Acadia University, Wolfville, Canada

2 Transportation Research Institute Hasselt University, Hasselt, Belgium

3 Weisberg Division of Computer Science, Marshall University, Huntington, WV, USA and features responsiveness, resiliency, and elasticity. The system is implemented using Scala that allowed the authors to reach a higher level of abstraction to be able to process any trajectory type. In this work, Geolife project's GPS trajectory dataset is utilized.

The second paper by Martins et al. is entitled "Improving Bluetooth Beacon-based Indoor Location and Fingerprinting". This paper investigated how Bluetooth Low Energy (BLE) beacons radio signals can be used for indoor location scenarios, as well as their precision. In this paper, a method for beacon-based positioning is introduced, which is based on signal strength measurements at key distances for each beacon. This method allowed the authors to use different beacon types, brands, and location conditions/constraints. Depending on each situation (i.e., hardware and location) it was possible to adapt the distance measuring curve to minimize errors and support higher distances, while at the same time keeping good precision. This paper also presented a comparison of their proposed approach with traditional positioning method, using formulas for distance estimation and the position triangulation. The proposed study is performed inside the campus of Viseu Polytechnic Institute, and tested using a group of students, where each student with his or her smart phone.

The third paper by Madani et al. is entitled "Finding Optimal Pedagogical Content in an Adaptive E-learning Platform using a New Recommendation Approach and Reinforcement Learning". This paper proposed a new recommendation approach for recommending relevant courses to learners. The proposed approach is based on social filtering using the notions of sentiment analysis) and collaborative filtering for defining the best way in which the learner must learn. As such, the system recommends courses that better match the learner's profile and social content. The authors of this work also proposed a new reinforcement learning approach to help learners to find the optimal learning path that can improve the quality of learning style and the results of the teaching process. 
The fourth paper by Mestoui et al. is entitled "Performance analysis of CE-OFDM-CPM Modulation using MIMO system over wireless channels". This this paper proposed a Constant Envelope OFDM (CE-OFDM) to provide a solution to the high Peak-to-Average Power Ratio (PAPR) issue in Orthogonal Frequency-Division Multiplexing (OFDM). In their approach, the OFDM signal is transformed, by way of Phase Modulation, to a constant envelope signal, thereby alleviating the need for a power back-off and allowing for the most efficient power amplifier operation possible. This paper also provided the implementation of Maximum-Likelihood Sequence Estimation channel equalization in CE-OFDM-PM. The authors found that a Multiple-Input Multiple-Output (MIMO) system showed better performance over wireless channels by providing communication links with substantial diversity and capacity. In this work, the simulation results showed that the Minimum Mean Square Error (MMSE) is the best one to avoid Inter-Symbol Interference.

The fifth paper by Kaja et al. is entitled "Acknowledgment Scheme using Cloud for Node Networks with EnergyAware Hybrid Scheduling Strategy". The authors described the substantial increase in the number of attacks in forever growing networks. This paper proposed an approach for acknowledgement scheme using cloud technology, called Cloud Acknowledgement Scheme. Their approach expected to strengthen the wireless network by introducing the cloud as a monitoring tool and to act as a leader node. The authors of this paper also developed algorithms to support the shortlived nature of acknowledgment packets in an efficient manner. The results revealed that the proposed approach has the potential to replace existing acknowledgement schemes.

The sixth paper by Ghai et al. is entitled "Efficient Algorithms to Minimize the End-to-End Latency of Edge Network Function Virtualization". This paper discussed the problem of minimizing the total latency for an edge network and how to efficiently solve it. The authors aimed to modify an existing latency minimization technique for edge Network Functions Virtualization (NFV) to make it more generic, using Integer Linear Programming (ILP). They applied a stable matching-based algorithm to solve the problem in polynomial time and then utilized a local search to improve its efficiency. The results showed the local search provided results that are close to optimal in a very reasonable time.

The seventh paper is Ragmani et al. is entitled "FACO: a hybrid fuzzy ant colony optimization algorithm for virtual machine scheduling in high-performance cloud computing". This paper proposed a hybrid fuzzy ant colony optimization algorithm (FACO) for virtual machine scheduling to guarantee high efficiency in a cloud environment. The contributions of this work include (1) the application of fuzzy logic to calculate the pheromones probability to minimize the computing time, and (2) the use of Taguchi experience design for selecting the best ACO's parameters to find the optimal parameters' combination. The demonstrated results using Cloud Analyst and CloudSim platforms showed their proposed approach improved load balancing in the Cloud architecture while reducing response time by up to $82 \%$, the processing time by up to $90 \%$, and the total cost by up to $9 \%$ depending on the applied scenario.

The eighth paper by Longhi et al. is entitled "Car Telematics Big Data Analytics for Insurance \& Innovative Mobility Services". The authors of this paper the importance of car telematics business sector that aims to collect mobility-related private and commercial vehicle data to develop services of various nature, both for individual citizens and other companies. These services and applications include information systems to support car insurance, info-mobility services, ad hoc studies for planning purposes, etc. In this work, the authors discussed selected key challenges that car telematics pilot application is facing within the EU project "Track and Know" and their appropriate solutions.

The guest editors would like to take this opportunity to thank all the authors for the efforts they put in the preparation of their manuscripts and for their valuable contributions. We wish to express our deepest gratitude to the referees, who provided valuable and thoughtful feedback to our authors. Our sincere thanks go to the Editor-in-Chief for his kind help and support. 